\title{
Accuracy analysis of high-order lattice Boltzmann models for rarefied gas flows
}

\author{
Jianping Meng and Yonghao Zhang \\ Department of Mechanical Engineering, University of Strathclyde, Glasgow G1 1XJ, UK
}

\begin{abstract}
In this work, we have theoretically analyzed and numerically evaluated the accuracy of high-order lattice Boltzmann (LB) models for capturing nonequilibrium effects in rarefied gas flows. In the incompressible limit, the LB equation is proved shown to be able to reduce to the linearized BhatnagarGross-Krook (BGK) equation. Therefore, when the same Gauss-Hermite quadrature is used, LB method closely assembles the discrete velocity method (DVM). In addition, the order of Hermite expansion for the equilibrium distribution function is found not to be directly correlated with the approximation order in terms of the Knudsen number to the BGK equation for incompressible flows. Furthermore, we have numerically evaluated the LB models for a standingshear-wave problem, which is designed specifically for assessing model accuracy by excluding the influence of gas molecule/surface interactions at wall boundaries. The numerical simulation results confirm that the high-order terms in the discrete equilibrium distribution function play a negligible role in capturing nonequilibrium effect for low-speed flows. Meanwhile, appropriate Gauss-Hermite quadrature has the most significant effect on whether LB models can describe the essential flow physics of rarefied gas accurately. For the same order of the Gauss-Hermite quadrature, the exact abscissae will also modestly influence numerical accuracy. Using the same Gauss-Hermite quadrature, the numerical results of both LB and DVM methods are in excellent agreement for flows across a broad range of the Knudsen numbers, which confirms that the LB simulation is similar to the DVM process. Therefore, LB method can offer flexible models
\end{abstract}


suitable for simulating continuum flows at the Navier Stokes level and rarefied gas flows at the linearized Boltzmann model equation level.

Keywords: Lattice Boltzmann method, computational fluid dynamics, non-equilibrium flow, gas microflow, standing-shear-wave problem

\section{Introduction}

Rarefied gas flows have recently attracted significant research interest due to the rapid development of micro/nano-fluidic technologies. Gaseous transport in micro/nano devices is often found to be non-equilibrium, and non-equilibrium phenomena have not yet been well understood[1]. The conventional theory to describe gas flows is the Navier Stokes equations, which assume that the fluid is in a quasi-equilibrium state. However, for non-equilibrium flows, the Navier Stokes equations break down because that the molecular nature of the gas strongly affects the bulk flow behavior, i.e., the gas can no longer be regarded as a fluid continuum. Whether gas flows are in local equilibrium or not can be classified by the non-dimensional Knudsen number, Kn, defined as the ratio of mean free path and the device characteristic length scale. The Navier Stokes equations with no-velocity-slip wall boundary condition are only appropriate when $K n<0.001$. However, gas flows in micro/nano-fluidic devices are often in the slip flow regime $(0.001<K n<0.1)$ or the transition flow regime $(0.1<$ $K n<10)$. In these regimes, the gas flow cannot be properly described as a continuous flow, nor as a free molecular flow. In practice, most devices operate with a range of Knudsen numbers in different parts of the device; this makes it even more difficult to develop a generalized flow model.

Direct simulation Monte Carlo (DSMC) methods and direct numerical simulation of the Boltzmann equation can provide accurate solutions for rarefied gas flows. However, these are computationally intractable for 3D flow systems, and impractical with the current computer technology, especially for the low speed gas flows usually encountered in micro/nano-systems[2]. Statistically, one needs to take significantly more samples of the flow field at any point for 
the DSMC method to resolve flows with low Mach numbers. The direct simulations based on the Boltzmann equation requires significant computational resources for integrating the velocity space ranging from $-\infty$ to $+\infty$. In addition, it is usually difficult to solve the full Boltzmann equation directly via either numerical or analytical methods.

Meanwhile, the continuum methods beyond the Navier Stokes level have failed to produce satisfactory results for gas flows in the transition flow regime [3]. It is well-known that continuum expressions for the viscous stress and heat flux in gases may be derived from the fundamental Boltzmann equation via either a Kn-series solution (known as the Chapman-Enskog approach) or by an expansion of the distribution function as a series of Hermite tensor polynomials[4]. To the first order (i.e., for near-equilibrium flows) both approaches can yield the Navier Stokes equations. However, the solution methods can be continued to second and higher orders, incorporating more and more of the salient characteristics of a non-equilibrium flow. The classical second-order stress and heat flux expressions are the Burnett equations (from the Chapman-Enskog approach), and the Grad 13-moment equations (from the Hermite polynomial method) [4]. These can be seen as corrections to the Navier Stokes constitutive relations to make them more appropriate to continuum-transition flows. However, different physical interpretations of the solution methods at second and higher orders have recently led to a variety of sets of equations, including the BhatnagarGross-Krook (BGK)-Burnett[5], Eu [6], augmented Burnett [7], and regularized moment (R13)equations [8]. While each purports to be the proper high-order correction to the stress and heat flux (there is no disagreement about the form of the Navier Stokes equations at the first-order), none of these models are is satisfactory [3]. In addition, these models suffer unknown additional boundary conditions at solid walls to appropriately reflect gas molecule/wall surface interactions.

The lattice Boltzmann (LB) approach may offer an alternative method for rarefied gas flow simulations. Historically, the LB model was evolved from the lattice-gas automata (LGA) for mimicking the Navier Stokes hydrodynamics, 
see Refs. $[9,10,11,12,13,14]$ and references therein. Over the past two decades, the LB method has been developed to provide accurate and efficient solutions for continuum flow simulations as the validity of the model can be ensured by the Chapman-Enskog expansion. Due to its kinetic nature, the LB model has distinct advantages over the continuum computational methods, including easy implementation of multi-physical mechanisms and the boundary conditions for fluid/wall interactions. In particular, the LB method is intended to seek a minimal set of velocities in phase space[10], which is an important feature different from traditional kinetic methods to effectively reduce the computational costs. The researchers have devoted significant efforts to extend the capability of LB models for rarefied gas flows, e.g., [15, 16, 17, 18, 19, 20, 21, 22, 23, 24, 25, 26, $27,28,29,30,31]$. In particular, the recently proposed high-order LB models have shown that the LB capability can go beyond the Navier-Stokes level $[19,21,22,24,26,27]$.

There are several methods to derive various order of LB models $[21,32,33$, $34,35,36,37,38,39]$. One of the most popular these approachs is based on the Hermite expansion[21, 32, 33, 34, 35, 38, 39], which creates another theoretical foundation different from the Chapman-Enskog expansion. Therefore, higher-order LB approximations to the BGK equation beyond the Navier Stokes level can be constructed by using high-order Hermite expansions with appropriate quadratures. This indicates that high-order LB models have the potential to capture non-equilibrium effects in rarefied flows. In addition to the systemic framework of constructing LB models, Shan et al. [21] also established the link between the orders of Hermite polynomials and Chapman-Enskog expansion and concluded that the order of Hermite expansion is responsible for obtaining correct velocity moments. The precise relation among the orders of Hermite expansion, Chapman-Enskog expansion and velocity moments was described by Eq.(4.7) in Ref.[21]. For instance, the third-order expansion is required for accurate pressure tensor and momentum at the Navier Stokes level. These conclusions are key to constructing appropriate LB models for non-equilibrium gas flows. However, the numerical simulations do not support these conclusions. In 
contrast, the simulation data showed that the higher order terms in the equilibrium distribution function have negligible influence for low speed rarefied flows [26]. This indicates that the Hermite expansion order is not directly related to the order of Chapman-Enksog expansion, in contrary to the theoretical conclusions drawn in Ref.[21].

In this work, we aim to answer this question whether the Hermite expansion order is important for the LB method, as it is for the Grad's moment method, to capture non-equilibrium effects in rarefied flows, especially at micro/nano-scales. Furthermore, we will analyze theoretically and numerically the mechanisms that are important in constructing high-order LB models for rarefied gas dynamics. We will discuss the differences between the approaches of Shan et al.[21] and Grad's moment method. To help us to understand the modeling capability of the LB method for rarefied gas dynamics, we will also analyze the similarities and differences between the LB method and the discrete velocity method (DVM) of solving the BGK equation. In particular, we will show that the Hermite expansion order for the equilibrium distribution function is not important for the flows that the linearized BGK equation can accurately describe. Since the important nonlinear constitutive relations in the Knudsen layer are still not captured satisfactorily[19], our numerical analysis will be based on a standingshear-wave problem specifically designed in Ref.[3] to exclude the effect of gas molecule/wall interactions, so we can concentrate on the model capabilities.

\section{Lattice Boltzmann simulation of rarefied gas flows}

\subsection{Lattice Boltzmann equation}

Although the LB models were originally developed from LGA, the link to the kinetic theory has later been established in Refs.[21, 32, 33, 34]. Consequently, the LB approach may be considered as a special finite difference scheme of solving the BGK equation. This theoretical link indicates that the LB methodology may provide a reasonable approximation to the BGK equation. The central question is how accurate the LB models can capture non-equilibrium effects in 
rarefied gas dynamics. To answer this question, we will revisit the derivation process of LB models from the BGK equation as described in Ref.[21] and we will emphasize on the model capability in describing rarefied gas flows.

The original BGK equation can be written as:

$$
\frac{\partial f}{\partial t}+\boldsymbol{\xi} \cdot \nabla f+\boldsymbol{g} \cdot \nabla_{\xi} f=-\frac{p}{\mu}\left(f-f^{e q}\right),
$$

where $f$ denotes the distribution function, $\boldsymbol{\xi}$ the phase velocity, $p$ the pressure, $\boldsymbol{g}$ the body force and $\mu$ the gas viscosity. Using the well-known Chapman-Enskog expansion, the collision frequency can be represented by the ratio of pressure and gas viscosity, which is convenient to obtain the Knudsen number definition consistent with that of hydrodynamic models. Without losing generality, we define the following non-dimensional variables:

$$
\begin{gathered}
\hat{\boldsymbol{r}}=\theta \boldsymbol{r}, \hat{\boldsymbol{u}}=\frac{\boldsymbol{u}}{\sqrt{R T_{0}}}, \hat{t}=\theta \sqrt{R T_{0}} t \\
\hat{\boldsymbol{g}}=\frac{\boldsymbol{g}}{\theta R T_{0}}, \hat{\phi}=\frac{\phi}{\theta \sqrt{R T_{0}}}, \hat{\boldsymbol{\xi}}=\frac{\boldsymbol{\xi}}{\sqrt{R T_{0}}}, \hat{T}=\frac{T}{T_{0}},
\end{gathered}
$$

where $\boldsymbol{u}$ is the macroscopic velocity, $R$ the gas constant, $T$ the gas temperature, $T_{0}$ the reference temperature, $\boldsymbol{r}$ the spatial position and $\theta$ the inverse of the characteristic length of the flow system. The symbol hat, which denotes a dimensionless value, will hereinafter be omitted. We define the Knudsen number using macroscopic properties as below:

$$
K n=\frac{\theta \mu \sqrt{R T_{0}}}{p} .
$$

By using these non-dimensional variables, the non-dimensional form of the BGK equation becomes

$$
\frac{\partial f}{\partial t}+\boldsymbol{\xi} \cdot \nabla f+\boldsymbol{g} \cdot \nabla_{\xi} f=-\frac{1}{K n}\left(f-f^{e q}\right),
$$

where the Maxwell distribution in $D$-dimensional Cartesian coordinates can be written as

$$
f^{e q}=\frac{\rho}{(2 \pi T)^{D / 2}} \exp \left[\frac{-(\boldsymbol{\xi}-\boldsymbol{u})^{2}}{2 T}\right]
$$


From the non-dimensional format of Eq.(4), we can clearly see the relationship between the relaxation time and the mean free path (i.e., Knudsen number), which plays a key role in LB simulation of rarefied gas flows, e.g., Ref.[15].

To discretize the velocity space, we project the distribution function onto a functional space spanned by the orthogonal Hermite basis:

$$
f(\boldsymbol{r}, \boldsymbol{\xi}, t) \approx f^{N}(\boldsymbol{r}, \boldsymbol{\xi}, t)=\omega(\boldsymbol{\xi}) \sum_{n=0}^{N} \frac{1}{n !} \boldsymbol{a}^{(n)}(\boldsymbol{r}, t) \boldsymbol{\chi}^{(n)}(\boldsymbol{\xi}),
$$

where $\boldsymbol{\chi}^{(n)}$ is the $n$th order Hermite polynomial. The weight function $\omega(\boldsymbol{\xi})$ is given by

$$
\omega(\boldsymbol{\xi})=\frac{1}{(2 \pi)^{D / 2}} \mathrm{e}^{-\xi^{2} / 2},
$$

and the coefficients $\boldsymbol{a}^{(n)}$ are

$$
\boldsymbol{a}^{(n)}=\int f \boldsymbol{\chi}^{(n)} d \boldsymbol{\xi} \approx \int f^{(N)} \chi^{(n)} d \boldsymbol{\xi}=\sum_{\alpha=1}^{d} \frac{w_{\alpha}}{\omega\left(\boldsymbol{\xi}_{\alpha}\right)} f^{(N)}\left(\boldsymbol{r}, \boldsymbol{\xi}_{\alpha}, t\right) \boldsymbol{\chi}^{(n)}\left(\boldsymbol{\xi}_{\alpha}\right) .
$$

The coefficient $a_{e q}^{(n)}$ for the equilibrium distribution is

$$
\boldsymbol{a}_{e q}^{(n)}=\int f^{e q} \boldsymbol{\chi}^{(n)} d \boldsymbol{\xi}
$$

where $w_{\alpha}$ and $\boldsymbol{\xi}_{\alpha}, a=1, \cdots, d$, are the weights and abscissae of a Gauss-Hermite quadrature of degree $\geq 2 N$ respectively. Herein, the distribution function is approximated by the first $N$ Hermite polynomial. Using the derivation relation, the body force term $F(\boldsymbol{r}, \boldsymbol{\xi}, t)=\boldsymbol{g} \cdot \nabla_{\xi} f$ can be approximated as

$$
F(\boldsymbol{r}, \boldsymbol{\xi}, t)=w \sum_{n=1}^{N} \frac{1}{(n-1) !} \boldsymbol{g} \boldsymbol{a}^{(n-1)} \chi^{(n)}\left(\boldsymbol{\xi}_{\alpha}\right) .
$$

As an example, the second order approximation of the equilibrium distribution and the body force are:

$$
\begin{gathered}
f^{e q} \approx \omega(\boldsymbol{\xi}) \rho\left\{1+\boldsymbol{\xi} \cdot \boldsymbol{u}+\frac{1}{2}\left[(\boldsymbol{\xi} \cdot \boldsymbol{u})^{2}-u^{2}+(T-1)\left(\xi^{2}-D\right)\right]\right\}, \\
F(\boldsymbol{r}, \boldsymbol{\xi}, t) \approx \omega(\boldsymbol{\xi}) \rho\{\boldsymbol{g} \cdot \boldsymbol{\xi}+(\boldsymbol{g} \cdot \boldsymbol{\xi})(\boldsymbol{u} \cdot \boldsymbol{\xi})-\boldsymbol{g} \cdot \boldsymbol{u}\},
\end{gathered}
$$


where $T$ should be set to unity for isothermal problems and $\rho$ is constant for incompressible problems.

An appropriate Gauss-Hermite quadrature, see the Appendix in Ref.[21] for a list of quadratures, can be chosen to evaluate the integral to obtain $a^{(n)}$. Consequently, Eq.(4) can be discretized as

$$
\frac{\partial f_{\alpha}}{\partial t}+\boldsymbol{\xi}_{\alpha} \cdot \nabla f_{\alpha}=-\frac{1}{K n}\left(f_{\alpha}-f_{\alpha}^{e q}\right)+g_{\alpha},
$$

where $f_{\alpha}=\frac{w_{\alpha} f\left(\boldsymbol{r}, \boldsymbol{\xi}_{\alpha}, t\right)}{\omega\left(\boldsymbol{\xi}_{\alpha}\right)}, f_{\alpha}^{e q}=\frac{w_{\alpha} f^{e q}\left(\boldsymbol{r}, \boldsymbol{\xi}_{\alpha}, t\right)}{\omega\left(\boldsymbol{\xi}_{\alpha}\right)}$ and $g_{\alpha}=\frac{w_{\alpha} F\left(\boldsymbol{r}, \boldsymbol{\xi}_{\alpha}, t\right)}{\omega\left(\boldsymbol{\xi}_{\alpha}\right)}$. We have obtained the lattice Boltzmann equation, i.e., Eq.(13), by discretizing Eq.(4) in the velocity space.

\subsection{Numerical scheme, Knudsen number and relaxation time}

An appropriate numerical scheme is now required to solve Eq.(13). If a finite difference scheme is chosen, one can obtain the so-called finite difference lattice Boltzmann model. In particular, when the first-order upwind finite-difference scheme is chosen, one can obtain the standard form of LB model. Herein, the following second-order numerical scheme for Eq.(13)[44, 31] is used.

$$
\begin{aligned}
f_{\alpha}\left(\boldsymbol{r}+\boldsymbol{\xi}_{\alpha} \delta t, t+\delta t\right)-f(\boldsymbol{r}, t)= & \frac{\delta t}{2 K n}\left[f_{\alpha}^{e q}\left(\boldsymbol{r}+\boldsymbol{\xi}_{\alpha} \delta t, t+\delta t\right)-f_{\alpha}\left(\boldsymbol{r}+\boldsymbol{\xi}_{\alpha} \delta t, t+\delta t\right)\right] \\
& -\frac{\delta t}{2 K n}\left[f_{\alpha}(\boldsymbol{r}, t)-f_{\alpha}^{e q}(\boldsymbol{r}, t)\right] \\
& +\frac{\delta t}{2}\left[g_{\alpha}\left(\boldsymbol{r}+\boldsymbol{\xi}_{\alpha} \delta t, t+\delta t\right)+g_{\alpha}(\boldsymbol{r}, t)\right] .
\end{aligned}
$$

By introducing

$$
\tilde{f}_{\alpha}=f_{\alpha}+\frac{\delta_{t}}{2 K n}\left(f_{\alpha}-f_{\alpha}^{e q}\right)-\frac{\delta_{t}}{2} g_{\alpha},
$$

the above implicit scheme can be written as

$$
\tilde{f}_{\alpha}\left(\boldsymbol{r}+\boldsymbol{\xi}_{\alpha} \delta_{t}, t+\delta_{t}\right)-\tilde{f}_{\alpha}(\boldsymbol{r}, t)=-\frac{\delta_{t}}{K n+0.5 \delta_{t}}\left[\tilde{f}_{\alpha}(\boldsymbol{r}, t)-f_{\alpha}^{e q}(\boldsymbol{r}, t)\right]+\frac{K n g_{\alpha} \delta_{t}}{K n+0.5 \delta_{t}},
$$

with

$$
\rho=\sum_{\alpha} \tilde{f}_{\alpha}
$$




$$
\rho \boldsymbol{u}=\sum_{\alpha} \boldsymbol{\xi}_{\alpha} \tilde{f}_{\alpha}+\frac{\rho \boldsymbol{g} \delta_{t}}{2}
$$

\subsection{High-order lattice Boltzmann models}

Although the construction of LB models based on the Hermite polynomials is straightforward, the Hermite polynomials higher than the third order give irrational roots. The integer stream velocity is an essential feature of LB models, i.e., the simple and efficient "stream-collision" mechanism. So high-order LB models, which have non-integer discrete velocities, will need additional effort, such as point-wise interpolation[45]. Therefore, they essentially become offlattice discrete velocity method for solving the kinetic Boltzmann equation, which will increase the computational cost dramatically and introduce extra numerical error. Shan et al.[21] suggested a method for searching abscissae on the grid points of Cartesian coordinates to construct high-order LB models with integer discrete velocities. The examples are D2Q17 and D2Q21 models given in Refs.[21] and [26] (note, we follow the conventional terminology for the LB models as first introduced in Ref.[9] dubbed as DnQm model, i.e., $\mathrm{n}$ dimensional model with $\mathrm{m}$ discrete velocities). Furthermore, Chikatamarla et al.[39] proposed an alternative method to seek rational-number approximation to the ratios of the Hermite roots based on the important relation between the entropy and the roots of Hermite polynomials. They also proposed the higherorder LB models with integer discrete velocity, such as D2Q16 and D2Q25 models. The above high-order LB models with integer stream velocities will be numerically examined in this work and the details are listed in Table.(1).

Based on the above model construction procedure, the accuracy of LB models depends on three level of approximations. Firstly, it depends on the accuracy of the numerical scheme for solving Eq.(13). As we have demonstrated, the commonly used first order upwind scheme will lead to incorrect physics for rarefied Hows. The second-order numerical scheme given by Eq. (16) is essential te eapture non-equilibrium effects accurately. Secondly, the order of the Hermite expansion was considered to be important to obtain the correct moments[21]. 


\begin{tabular}{|c|c|c|c|c|}
\hline Quadrature & $\mathrm{k}$ & $\xi_{\alpha}$ & $w_{\alpha}$ & \\
\hline \multirow[t]{3}{*}{ D2Q9 } & 1 & $(0,0)$ & $4 / 9$ & \\
\hline & 4 & $(\sqrt{3}, 0)_{F S}$ & $1 / 9$ & \\
\hline & 4 & $( \pm \sqrt{3}, \pm \sqrt{3})$ & $1 / 36$ & \\
\hline \multirow[t]{4}{*}{ D2Q16 } & 4 & $( \pm m, \pm m)$ & $W_{ \pm m}^{2}$ & $m=1, n=4$ \\
\hline & 4 & $( \pm n, \pm n)$ & $W_{ \pm n}^{2}$ & $W_{ \pm m}=\frac{m^{2}-5 n^{2}+\sqrt{m^{4}-10 n^{2} m^{2}+n^{4}}}{12\left(m^{2}-n^{2}\right)}$ \\
\hline & 4 & $( \pm m, \pm n)$ & $W_{ \pm m} W_{ \pm n}$ & $W_{ \pm n}=\frac{5 m^{2}-n^{2}-\sqrt{m^{4}-10 n^{2} m^{2}+n^{4}}}{12\left(m^{2}-n^{2}\right)}$ \\
\hline & 4 & $( \pm n, \pm m)$ & $W_{ \pm m} W_{ \pm n}$ & $T_{0}=\left(m^{2}+n^{2}+\sqrt{m^{4}-10 n^{2} m^{2}+n^{4}}\right) / 6$ \\
\hline \multirow[t]{5}{*}{ D2Q17 } & 1 & $(0,0)$ & $\frac{575+193 \sqrt{193}}{8100}$ & \\
\hline & 4 & $(r, 0)_{F S}$ & $\frac{3355-91 \sqrt{193}}{18000}$ & $r^{2}=(125+5 \sqrt{193}) / 72$ \\
\hline & 4 & $( \pm r, \pm r)$ & $\frac{655+17 \sqrt{193}}{27000}$ & \\
\hline & 4 & $( \pm 2 r, \pm 2 r)$ & $\frac{685-49 \sqrt{193}}{54000}$ & \\
\hline & 4 & $(3 r, 0)_{F S}$ & $\frac{1445-101 \sqrt{193}}{162000}$ & \\
\hline \multirow[t]{6}{*}{$\mathrm{D} 2 \mathrm{Q} 21$} & 1 & $(0,0)$ & $91 / 324$ & \\
\hline & 4 & $(r, 0)_{F S}$ & $1 / 12$ & $r^{2}=3 / 2$ \\
\hline & 4 & $( \pm r, \pm r)$ & $2 / 27$ & \\
\hline & 4 & $( \pm 2 r, 0)_{F S}$ & $7 / 360$ & \\
\hline & 4 & $( \pm 2 r, \pm 2 r)$ & $1 / 432$ & \\
\hline & 4 & $(3 r, 0)_{F S}$ & $1 / 1620$ & \\
\hline \multirow[t]{7}{*}{ D2Q25 } & 1 & $(0,0)$ & $W_{0}^{2}$ & $m=3, n=7$ \\
\hline & 4 & $(m, 0) F S$ & $W_{ \pm m} W_{0}$ & $W_{0}=\frac{-3 m^{4}-3 n^{4}+54 m^{2} n^{2}-\left(m^{2}+n^{2}\right) D_{5}}{75 m_{2}^{2} n^{2}}$ \\
\hline & 4 & $(n, 0)_{F S}$ & $W_{ \pm n} W_{0}$ & $W_{ \pm m}=\frac{9 m^{4}-6 n^{4}-27 n^{2} m^{2}+\left(3 m^{2}-2 n^{2}\right) D_{5}}{300 m^{2}\left(m^{2}-n^{2}\right)}$ \\
\hline & 4 & $( \pm m, \pm m)$ & $W_{ \pm m}^{2}$ & $W_{ \pm n}=\frac{9 n^{4}-6 m^{4}-27 n^{2} m^{2}+\left(3 n^{2}-2 m^{2}\right) D_{5}}{300 n^{2}\left(n^{2}-m^{2}\right)}$ \\
\hline & 4 & $( \pm n, \pm n)$ & $W_{ \pm n}^{2}$ & $T_{0}=\left(3 m^{2}+3 n^{2}+D_{5}\right) / 30$ \\
\hline & 4 & $( \pm m, \pm n)$ & $W_{ \pm m} W_{ \pm n}$ & $D_{5}=\sqrt{9 m^{4}-42 n^{2} m^{2}+9 n^{4}}$ \\
\hline & 4 & $( \pm n, \pm m)$ & $W_{ \pm m} W_{ \pm n}$ & \\
\hline
\end{tabular}

Table 1: The quadratures of five LB models where $\mathrm{k}$ is the number of discrete velocities with the same velocity magnitude, the subscript $F S$ denotes a fully symmetric set of points, and $w_{\alpha}$ are the weights. The quadrature accuracy is fifth-order for the D2Q9 model, seventh-order for the D2Q16, D2Q17 and D2Q21 models, and ninth-order for the D2Q25 model. The details of D2Q17 and D2Q21 models can be found in Refs.[21, 26] while the D2Q16 and D2Q25 models are discussed in Ref.[39].

Thirdly, the Gauss-Hermite quadrature accuracy should be sufficiently high so that the integration of Eq.(8) can be evaluated accurately. Therefore, the term higher-order LB models here refer to the LB models with high-order of Hermite expansion and Gauss-Hermite quadrature in comparison with the standard LB model. 


\section{Lattice Boltzmann, moment and discrete velocity method}

\subsection{Lattice Boltzmann method and Grad's moment method}

Similar to the Grad's method for deriving higher order continuum systems (e.g, Grad 13-moment equations), using the Hermite expansion to approximate the BGK equation can lead to the LB equation, i.e., Eq.(13). However, the major difference is that LB models are always staying at the kinetic level, i.e., solving the kinetic equation - Eq.(13), while the Grad's method will produce a set of continuum equations. The central idea of Grad's method is to use the truncated Hermite polynomials to approximate the full Boltzmann or BGK equation. Due to the unique feature of Hermite polynomial, the moments of up to the chosen truncation order can be described accurately by the derived macroscopic moments systems. In contrary, the only explicit effect of the truncation on the LB models is on the approximation of the equilibrium distribution function and the body force, while the Grad's moment equations do not approximate the equilibrium distribution function.

Although the order of Hermite expansion determines the accuracy level of the moment model, which is not the same for the LB models. Essentially, the LB equation, i.e., Eq.(13) is similar to any model equation which is to simplify the full Boltzmann equation. The kinetic process, i.e., gas molecules relaxing to the equilibrium state through collisions, is still the same. Therefore, the LB method is very close to the discrete velocity method solving the BGK equation (especially the linearized-BGK equation), which we will discuss in the section below.

\subsection{Lattice Boltzmann method and discrete velocity method}

The above procedure of establishing LB models is similar to the problem solving process of the discrete velocity method, which directly solves the BGK equation. Indeed, the relation to DVM was already recognized in late 1990s[32, $33,34,21]$. Since DVM has been proved to be able to provide accurate results for rarefied gas dynamics, see Refs.[46, 47, 48, 49, 50, 51, 52, 53, 54, 55, 56] and 
references therein, it is helpful to further compare two numerical methods in depth.

The discrete velocity method is to discretize the velocity space based on quadratures, e.g., Gauss-Hermite quadrature and Newton-Cotes quadrature, see Refs. $[54,53,52,49]$. The first step is to non-dimensionalize the BGK equation and obtain the reduced functions, which are important to reduce computational costs. The second step is to apply an appropriate discretization method for the velocity space, which is important but difficult because the velocity space ranges from $-\infty$ to $+\infty$ and the properties of conservation and dissipation of the entropy should be kept. A typical choice is the Gauss-Hermite quadrature, which is to be adopted in our simulations. In order to reduce the velocity components which need to be integrated from $-\infty$ to $+\infty$, curvilinear coordinates, e.g., the polar coordinates for 2D systems may be used for the velocity space. Afterwards, the continuous Maxwell equilibrium should also be discretized. The last step is to adopt an appropriate numerical scheme for the space and time discretization. Therefore, we can see that LB methodology closely resembles the DVM problem solving process. It is essentially DVM with finite discrete velocities and fully discretized space and time tied to the discrete velocity set[57]. For simulating rarefied gas flows, this similarity is important as we have shown how the LB framework is developed from the BGK equation.

For both DVM and LB methods, the most critical task is to discretize the velocity space. When the Gauss-Hermite quadrature is used in DVM, the discretization of the velocity space in these two methods are the same, which may indicate that the LB models with sufficiently accurate Gauss-Hermite quadrature can capture the higher-order non-equilibrium effects in the rarefied gas flows. This indeed is confirmed by the simulation results presented in Fig.1, which we will discuss in detail in Section 4.

However, as stated by Chen et al.[10], an unique feature of the LB methodology different from traditional kinetic methods is devoting efforts to seek a minimal discrete velocity set according to the required accuracy. Moreover, the "stream-collision" mechanism, which is mainly inherited from the lattice gas 
automata, makes the LB method easy to understand and efficient to computing. Therefore, the LB methodology is conceptually straightforward and more suitable for developing a generic engineering-purpose package, e.g., Powerflow ${ }^{1}$. It is worthwhile to extend the capability of the LB methodology to simulate non-equilibrium flows in many industrial relevant applications. By contrary, current DVM approach relies heavily on mathematical techniques which are chosen for specific problems and emphasizes on model accuracy at cost of computational efficiency. In particular, DVM needs more research efforts in near hydrodynamics flow regime. Therefore, by combining the unique features of LBM and DVM, appropriate models can be designed to achieve the required accuracy with minimal computational effort.

\subsection{Lattice Boltzmann equation and linearized BGK equation}

By introducing $\psi$ to denote the unknown perturbed distribution function and assuming the flow is not far from equilibrium, $f$ can be approximated by

$$
f=f^{0}(1+\psi),
$$

where

$$
f^{0}=\frac{1}{(2 \pi)^{D / 2}} \mathrm{e}^{-\xi^{2} / 2},
$$

which is the global (absolute) equilibrium distribution function. Using the Taylor series to expand the local equilibrium distribution function and keeping the terms up to the first order, one can obtain the following equation

$$
\frac{\partial f}{\partial t}+\boldsymbol{\xi} \cdot \nabla f+\boldsymbol{g} \cdot \nabla_{\xi} f=-\frac{1}{K n}\left\{f-f^{0}\left[1+\boldsymbol{\xi} \cdot \boldsymbol{u}+\frac{1}{2}(T-1)\left(\xi^{2}-D\right)\right]\right\},
$$

where we assume the flow is incompressible. Using Eq.(19), we can obtain the linearized BGK equation:

$$
\left.\frac{\partial \psi}{\partial t}+\boldsymbol{\xi} \cdot \nabla \psi+\boldsymbol{g} \cdot\left[\nabla_{\xi} \psi-(1+\psi) \boldsymbol{\xi}\right)\right]=-\frac{1}{K n}\left\{\psi-\left[\boldsymbol{\xi} \cdot \boldsymbol{u}+\frac{1}{2}(T-1)\left(\xi^{2}-D\right)\right]\right\} .
$$

\footnotetext{
${ }^{1}$ see http://www.exa.com/
} 
For lattice Boltzmann models, one can rewrite Eq.(6) as

$$
f(\boldsymbol{r}, \boldsymbol{\xi}, t) \approx f^{N}(\boldsymbol{r}, \boldsymbol{\xi}, t)=\omega(\boldsymbol{\xi})[1+\varphi(\boldsymbol{r}, \boldsymbol{\xi}, t)],
$$

where

$$
\varphi(\boldsymbol{r}, \boldsymbol{\xi}, t)=\sum_{n=1}^{N} \frac{1}{n !} \boldsymbol{a}^{(n)}(\boldsymbol{r}, t) \boldsymbol{\chi}^{(n)}(\boldsymbol{\xi}) .
$$

Substituting Eq.(23) into the BGK equation and keeping the first- and secondorder expansions of the equilibrium distribution, one can obtain

$$
\frac{\partial \varphi}{\partial t}+\boldsymbol{\xi} \cdot \nabla \varphi+\boldsymbol{g} \cdot\left[\nabla_{\xi} \varphi-(1+\varphi) \boldsymbol{\xi}\right]=-\frac{1}{K n}(\varphi-\boldsymbol{\xi} \cdot \boldsymbol{u}),
$$

and

$$
\begin{aligned}
\frac{\partial \varphi}{\partial t}+\boldsymbol{\xi} \cdot \nabla \varphi+\boldsymbol{g} \cdot\left[\nabla_{\xi} \varphi-(1+\varphi) \boldsymbol{\xi}\right] & =-\frac{1}{K n}\{\varphi-\boldsymbol{\xi} \cdot \boldsymbol{u} \\
& \left.-\frac{1}{2}\left[(\boldsymbol{\xi} \cdot \boldsymbol{u})^{2}-u^{2}+(T-1)\left(\xi^{2}-D\right)\right]\right\}
\end{aligned}
$$

Because $\omega(\xi)$ is equal to $f_{0}$, we can observe the following interesting facts by comparing Eqs.(25) and (26) with Eq.(22). First of all, by keeping the first order Hermite expansion, the essential LB model equation is the same with that of the isothermal $(T=1)$ linearized BGK equation except the body force term. This implies that $\varphi$ is indeed equivalent to $\psi$ though $\varphi$ is prescribed to include only the finite order terms of the Hermite polynomials (cf. Eq.(6) and Eq.(23)). Therefore, the LB equation with the first order terms should be as good as the linearized BGK equation for isothermal flows. This indicates that high-order Hermite expansion for equilibrium distribution function is not necessary for low-speed rarefied gas flows. Secondly, with the second order Hermite expansion, there is an extra velocity term $\frac{1}{2}\left[(\boldsymbol{\xi} \cdot \boldsymbol{u})^{2}-u^{2}\right]$ for the LB equation in comparison to the linearized BGK equation. However, for flows with low Mach number, this term is a higher-order small quantity which can be ignored. This is the reason why the Hermite expansion order is reported to make negligible difference on the simulation results[26]. In fact, the first order expansion is sufficient to obtain the accurate results for isothermal rarefied flows with low speed. Furthermore, the LB equation with the second order expansion 
can in principle describe thermal problems since the temperature information is included in Eq.(26), which at least has the same capability as the linearized BGK equation, though the BGK kinetic model gives wrong Pr number. Thirdly, the treatment of the body force makes the difference between the LB model and the linearized BGK equation. It is because that the linearized BGK model keeps the full information while the LB model uses the Hermite expansion to approximate $\nabla_{\xi} f$, i.e., $\nabla_{\xi} \varphi-(1+\varphi) \boldsymbol{\xi}$. However, for the problem is not far from equilibrium state, this difference is not important, which will be confirmed by the numerical simulations in Section 4 .

From the above analysis, we can see that the Hermite expansion order does not directly determine the accuracy of LB models for rarefied gas flows as described by Eq.(4.7) in Ref.[21]. The Hermite expansion provides a means to approximate the equilibrium distribution and the body force in the kinetic equation. Therefore, the LB equation, similar to the linearized BGK equation, is an approximation of the BGK equation. In contrast to the Grad's moment method, LB models include the information of any order moment though it may not be accurate. For instance, with the first order expansion, the LB model equation is as the same as the isothermal linearized BGK equation in the incompressible limit, which will give accurate results for any order velocity moment. When the Mach number of flow increases, high-order terms in the Hermite expansion become important $[58,59]$. Therefore, the order of Hermite expansion is important to simulate compressible flows rather than rarefied flows.

To capture non-equilibrium effects in rarefied flows, the Gauss-Hermite quadrature is the key as it determines the discretization accuracy to the model equation. With sufficiently high order of the Gauss-Hermite quadrature, LB models can give excellent numerical results, e.g., the results presented in Fig.(1) where 400 discrete velocities are used are identical to the DVM solution. Considering the similarity of the LB equation and linearized BGK equation, insufficient quadrature order should be responsible for the failure on capturing the constitutive relations in the Knudsen layer because the kinetic boundary condition have been well accepted in solving the linearized BGK equation. 
In summary, the LB method approximates the BGK equation with finite discrete velocities and fully discretized space and time tied to the discrete velocity set. The capability of LB equation is similar to the linearized BGK equation for simulating rarefied gas flows in the incompressible limit. The Hermite expansion order determines the model equation and is important for compressible flows. It has no direct effect on the accuracy of capturing high-order non-equilibrium effects. Meanwhile, the Gauss-Hermite quadrature as a discretization technique for the velocity space directly determines whether the LB models can describe rarefied flows accurately.

\section{Simulations and discussion}

In addition to the above theoretical analysis, we will numerically evaluate the LB models. To exclude the boundary condition effect, we choose the standingshear-wave problem as the benchmark case, which was specially designed for assessing the accuracy of various models for isothermal flows[3]. It is a shear flow driven by a temporally and spatially oscillating body force, which can be written as the following form:

$$
F_{x}=A \mathrm{e}^{\mathrm{i} \phi t} \cos \theta y
$$

where $F_{x}$ is the body force in the direction $x$ (which is perpendicular to the $y$ direction), $A$ is the amplitude, $\theta$ is the wave number, and $\phi$ is the frequency. This isothermal problem is sufficiently simple because the flow direction is perpendicular to the space variation but it is intended to capture the shear-dominated characteristic of microscale flows. Furthermore, the distinct advantage is that the boundary is not important here so that one can focus on the model itself without the interference from gas molecule/wall interactions. With Eq.(2), the body force becomes:

$$
F_{x}=\hat{A} \mathrm{e}^{\mathrm{i} \phi t} \cos y,
$$

where $\theta$ is considered as a measure of the characteristic length, and $\hat{A}=\frac{\boldsymbol{A}}{\theta R T}$. Another distinctive advantage for using this benchmark problem is that ana- 
lytical solutions can be obtained for many hydrodynamic models, such as the Navier Stokes equation and the regularized 13-moment model (R13).

Fig. 1 shows that, as an extreme test case, the LB model with 400 discrete velocities can obtain results for velocity amplitude nearly identical to the linezrized BGK equation for a broad range of Knudsen numbers from 0.1 to 1.5, which confirms the capability of the LB equations. Since only the first Hermite expansion is used, it indicates that the Hermite expansion order does not directly affect accuracy of the LB models in capturing non-equilibrium effects measured by the Knudsen number for incompressible flows.

Although the standard LB model (D2Q9) is not sufficiently accurate in comparison with the DVM solution, high-order LB model (D2Q16) with minimal increase of the discrete velocity set can produce good results. Fig.1 shows that the LB model with increasing order of the Gauss-Hermite quadrature can closely approximate the linearized BGK equation. Therefore, in comparison with the DVM simulation, LB method can provide a practical engineering design simulation tool which can produce reasonably accurate results with significantly reduced computational cost.

As discussed in Section 2.3, at least three factors will influence the problemsolving process, i.e., the numerical scheme for solving Eq.(13), the order of Hermite expansion and Gauss-Hermite quadrature. For the numerical scheme, the second-order scheme is essential as discussed in Section 2.2. Regarding the role of Hermite expansion and Gauss-Hermite quadrature, we have theoretically shown that the Gauss-Hermite quadrature rather than the order of Hermite expansion is key to capturing non-equilibrium effects accurately. The numerical simulations have also performed to testify our theoretical analysis.

In Figs.2 and 3, the simulation results of the three LB models are compared with the solutions of directly solving the linearized BGK equation and the Navier Stokes equation. The expansion of the equilibrium distribution function and the forcing term is second-order for the D2Q9 model, third-order for the D2Q16 and forth-order for D2Q25. The results in Fig.2 show that the prediction for velocity amplitude of the D2Q25 model are in excellent agreement with the 
DVM solution of the linearized BGK equation across a broad range of Knudsen number $(K n \in[0,1.5])$ for the quasi-steady and time-varying problems with $\theta$ up to 0.25. Meanwhile, the results of the D2Q9 model deviate from the DVM solution of the linearized BGK equation significantly. Surprisingly, the D2Q9 model does not agree with the results predicted by the Navier Stokes equation. Fig.3 shows the velocity wave phase lag and shear pressure amplitude, which suggests that high-order LB models perform better in the transition flow regime.

Although Figs.2 and 3 demonstrate that increasing order of LB model in terms of the Hermite expansion and Gauss-Hermite quadrature will lead to more accurate results, we still do not know the exact role the orders of the Hermite expansion and the Gauss-Hermite quadrature play. Therefore, we single out the effect of the Hermite expansion in Fig.4, where the results of the LB models with the same quadrature but different Hermite expansion order are compared. The results clearly show that the Hermite expansion order for both the force and the equilibrium distribution function make negligible difference to the simulation results. Even the first order expansion is sufficient to obtain accurate velocity for the D2Q25 model. The simulation results support our theoretical analysis that the Hermite expansion has no direct influence on model accuracy for capturing non-equilibrium effects. Specifically, the LB model equation determined by the first order Hermite expansion is sufficient for a typical gas flow in microdevices where the Mach number is usually small. In contrast, the Gauss-Hermite quadrature determines the model accuracy as the higher-order quadratures give better results.

Not only the order of quadrature but also the abscissae may influence the model accuracy. Therefore, the simulation results of the three LB models with the same order quadrature but different abscissae are compared in Fig.5. Although increasing quadrature order will lead to improved accuracy, more discrete velocities may not improve the model performance if the quadratures are the same order. For example, the quadratures of the D2Q16, D2Q17 and D2Q21 models are the same order. Surprisingly, the D2Q16 model produces the results better than the other two models with more discrete velocities. The reason may 
be attributed to that the abscissae of the D2Q16 model has better symmetry. In addition, all these models are better than the D2Q9 model which has low order quadrature. Therefore, appropriate abscissae may improve the model accuracy and reduce the computational costs with smaller number of discrete velocities.

Since it has been shown in Ref.[3] that the R13 equation gives the best performance among the extended hydrodynamic models, we compare the LB models with the R13 model here. Fig. 6 shows that, in comparison with the data obtained from directly solving the linearized BGK equation, the high-order LB models including the D2Q16 and D2Q25 models can give better results than the R13 equation over a broad range of Knudsen numbers. Therefore, the high-order LB models with modest number of discrete velocity set, such as the D2Q16 and D2Q25 models, can offer close approximation to the linearized BGK equation. Most importantly, these high-order LB models achieve such degree of accuracy at a fraction of computational costs associated with directly solving the linearized BGK equation.

It will be interesting to compare the computational costs associated with different quadratures. Here, the comparison is between the D2Q25 and D2Q400 models for a quasi-steady standing shear wave problem, and the simulations are performed with a four-core PC (Intel Core 2 Quad Q6600@2.4GHz). The first order expansion of the equilibrium distribution function is used for both models. The convergence criterion is set as $\left|\frac{\bar{u}^{n+1}-\bar{u}^{n}}{\bar{u}^{n}}\right|<10^{-08}$, where $\bar{u}^{n+1}$ and $\bar{u}^{n}$ are the velocity amplitude at the consecutive steps. We test the simulations with the same grid numbers at different Knudsen numbers. When $K n=0.5$, the D2Q400 model needs about 85 minutes (23647 iterations) to obtain the simulation results, while the D2Q25 only needs about 21 seconds (3882 iterations). For the flow with $K n=0.7$, the D2Q400 needs about 66 minutes (18376 iterations), while the D2Q25 model needs about 14 seconds (2692 iterations). It is worth noting that the difference of simulation results between these two models is less than $3 \%$. 


\section{Concluding remarks}

We have theoretically and numerically analyzed the high-order LB models for rarefied gas flows. The lattice Boltzmann equation is shown to be able to reduce to the linearized BGK equation in the incompressible limit. When the same Gauss-Hermite quadrature is used, both LB and DVM simulations produce results in excellent agreement across a broad range of the Knudsen numbers. This suggests the importance of the Gauss-Hermite quadrature and the great potential of the LB method for modeling rarefied gas flows. While the Gauss-Hermite quadrature is of the most importance to capturing non-equilibrium effects, the first-order Hermite expansion on the equilibrium distribution function is sufficient to obtain the correct moments for isothermal flows, e.g., increasing the Hermite expansion order further will not improve the model accuracy in the incompressible limit. For the same order Gauss-Hermite quadratures, the chosen abscissae will influence the model accuracy and more discrete velocities may not lead to improved model accuracy.

Overall, we have demonstrated that LB method offers a computationally efficient approach to solve the BGK equation. We can choose a suitable LB model to meet different requirement on model accuracy and computational efficiency, which offers an ideal flexible engineering design simulation tool to be able to simulate flows in the continuum and transition regimes.In particular, this work may lay down the foundation for developing a multiscale LB model for mix knudsen number flows, which can be a specific method for the unified kinetic scheme as shown by Ref.[60]

\section{Acknowledgments}

The authors would like to thank Jason Reese, Xiaojun Gu, Alan Keir and Guihua Tang for their critical comments and technical help. This work was financially supported by the Engineering and Physical Sciences Research Council U.K. under Grants No. EP/ F028865/1. The research leading to these results has received the funding from the European Community's Seventh Frame- 
work Programme FP7/2007-2013 under grant agreement ITN GASMEMS No. 215504 .

[1] C. M. Ho, Y. C. Tai, Micro-electro-mechanical-systems (MEMS) and fluid flows, Annu. Rev. Fluid Mech. 30 (1) (1998) 579-612.

[2] J. Reese, M. Gallis, D. Lockerby, New directions in fluid dynamics: nonequilibrium aerodynamic and microsystem flows, Phil. Trans. R. Soc. Lond. A 361 (2003) 2967-2988.

[3] D. Lockerby, J. Reese, On the modelling of isothermal gas flows at the microscale, J. Fluid Mech. 604 (2008) 235-261.

[4] S. Chapman, T. G. Cowling, The Mathematical Theory of Non-uniform Gases, Cambridge University Press., 1991.

[5] R. Balakrishnan, An approach to entropy consistency in second-order hydrodynamic equations, J. Fluid Mech. 503 (2004) 201-245.

[6] M. Al-Ghoul, B. Chan Eu, Generalized hydrodynamics and microflows, Phys. Rev. E 70 (1) (2004) 016301.

[7] X. L. Zhong, D. R. Chapman, R. W. MacCormack, Stabilization of the Burnett equations and application to hypersonicflows, AIAA Journal 31 (1993) 1036-1043.

[8] H. Struchtrup, M. Torrilhon, Regularization of Grad's 13 moment equations: Derivation and linear analysis, Phys. Fluids 15 (9) (2003) 2668-2680.

[9] Y. H. Qian, D. D'Humières, P. Lallemand, Lattice BGK models for NavierStokes equation, Europhys. Lett. 17 (6) (1992) 479-484.

[10] S. Y. Chen, G. D. Doolen, Lattice Boltzmann method for fluid flows, Annu. Rev. Fluid Mech. 30 (1998) 329-364.

[11] R. Benzi, S. Succi, M. Vergassola, The lattice Boltzmann equation: Theory and applications, Phys. Rep. 222 (1992) 145-197. 
[12] S. Succi, I. V. Karlin, H. D. Chen, Colloquium: Role of the H theorem in lattice Boltzmann hydrodynamic simulations, Rev. Mod. Phys. 74 (4) (2002) 1203-1220.

[13] Y. H. Qian, S. Succi, S. Orszag, Recent advances in lattice Boltzmann computing, Annu. Rev. Comput. Phys. III (1995) 195-242.

[14] C. K. Aidun, J. R. Clausen, Lattice-Boltzmann method for complex flows, Annu. Rev. Fluid Mech. 42 (1) (2010) 439-472.

[15] Y. H. Zhang, R. S. Qin, D. R. Emerson, Lattice Boltzmann simulation of rarefied gas flows in microchannels, Phys. Rev. E 71 (4) (2005) 047702.

[16] F. Toschi, S. Succi, Lattice Boltzmann method at finite Knudsen numbers, Europhys. Lett. 69 (4) (2005) 549-555.

[17] M. Sbragaglia, S. Succi, Analytical calculation of slip flow in lattice Boltzmann models with kinetic boundary conditions, Phys. Fluids 17 (9) (2005) 093602.

[18] M. Sbragaglia, S. Succi, A note on the lattice Boltzmann method beyond the Chapman-Enskog limits, Europhys. Lett. 73 (3) (2006) 370-376.

[19] G. H. Tang, Y. H. Zhang, D. R. Emerson, Lattice Boltzmann models for nonequilibrium gas flows, Phys. Rev. E 77 (4) (2008) 046701.

[20] Y. H. Zhang, X. J. Gu, R. W. Barber, D. R. Emerson, Capturing Knudsen layer phenomena using a lattice Boltzmann model, Phys. Rev. E 74 (4) (2006) 046704.

[21] X. W. Shan, X. F. Yuan, H. D. Chen, Kinetic theory representation of hydrodynamics: A way beyond the Navier Stokes equation, J. Fluid Mech. 550 (2006) 413-441.

[22] R. Y. Zhang, X. W. Shan, H. D. Chen, Efficient kinetic method for fluid simulation beyond the Navier-Stokes equation, Phys. Rev. E 74 (4) (2006) 046703. 
[23] I. V. Karlin, S. Ansumali, Renormalization of the lattice Boltzmann hierarchy, Phys. Rev. E 76 (2007) 025701(R).

[24] S. Ansumali, I. V. Karlin, S. Arcidiacono, A. Abbas, N. I. Prasianakis, Hydrodynamics beyond Navier-Stokes: Exact solution to the lattice Boltzmann hierarchy, Phys. Rev. Lett. 98 (12) (2007) 124502.

[25] S. H. Kim, H. Pitsch, I. D. Boyd, Slip velocity and Knudsen layer in the lattice Boltzmann method for microscale flows, Phys. Rev. E. 77(2008)026704.

[26] S. H. Kim, H. Pitsch, I. D. Boyd, Accuracy of higher-order lattice Boltzmann methods for microscale flows with finite Knudsen numbers, J. Comput. Phys. 227 (19) (2008) 8655 - 8671.

[27] W. P. Yudistiawan, S. Ansumali, I. V. Karlin, Hydrodynamics beyond Navier-Stokes: The slip flow model, Phys. Rev. E 78 (1) (2008) 016705.

[28] L. Zheng, Z. L. Guo, B. C. Shi, Discrete effects on thermal boundary conditions for the thermal lattice Boltzmann method in simulating microscale gas flows, Europhys. Lett. 82 (2008) 44002.

[29] Z. L. Guo, B. C. Shi, T. S. Zhao, Discrete effects on boundary conditions for the lattice Boltzmann equation in simulating microscale gas flows, Phys. Rev. E, 76 (2007) 056704.

[30] Z. W. Tian, C. Zhou, H. J. Liu, Z. L. Guo, Z. H. L, C. G. Zheng, Lattice Boltzmann scheme for simulating thermal micro-flow, Physica A, 385 (2007) $59-68$.

[31] Z. L. Guo, T. S. Zhao, Y. Shi, Physical symmetry, spatial accuracy, and relaxation time of the lattice Boltzmann equation for microgas flows, J. Appl. Phys., 99 (2005) 074903.

[32] X. Y. He, L. S. Luo, A priori derivation of the lattice Boltzmann equation, Phys. Rev. E 55 (6) (1997) R6333-R6336. 
[33] X. Y.He, L. S. Luo, Theory of the lattice Boltzmann method: From the Boltzmann equation to the lattice Boltzmann equation, Phys. Rev. E 56 (6) (1997) 6811-6817.

[34] X. W. Shan, X. Y. He, Discretization of the velocity space in the solution of the Boltzmann equation, Phys. Rev. Lett. 80 (1) (1998) 65-68.

[35] N. S. Martys, X. W. Shan, H. D. Chen, Evaluation of the external force term in the discrete Boltzmann equation, Phys. Rev. E 58 (5) (1998) 68556857.

[36] I. V. Karlin, A. Ferrante, H. C. Öttinger, Perfect entropy functions of the lattice Boltzmann method, Europhys. Lett. 47 (2) (1999) 182-188.

[37] S. Ansumali, I. V. Karlin, Stabilization of the lattice Boltzmann method by the H theorem: A numerical test, Phys. Rev. E 62 (6) (2000) 7999-8003.

[38] S. Ansumali, I. V. Karlin, H. C. Öttinger, Minimal entropic kinetic models for hydrodynamics, Europhys. Lett. 63 (6) (2003) 798-804.

[39] S. S. Chikatamarla, I. V. Karlin, Entropy and galilean invariance of lattice Boltzmann theories, Phys. Rev. Lett. 97 (19) (2006) 190601.

[40] M. B. Reider, J. D. Sterling, Accuracy of discrete-velocity BGK models for the simulation of the incompressible Navier-Stokes equations, Comput. Fluids 24 (4) (1995) $459-467$.

[41] J. D. Sterling, S. Y. Chen, Stability analysis of lattice Boltzmann methods, J. Comput. Phys. 123 (1) (1996) 196-206.

[42] X. B. Nie, G. D. Doolen, S. Y. Chen, Lattice-Boltzmann simulations of fluid flows in MEMS, J. Stat. Phys. 107 (1) (2002) 279-289.

[43] C. Y. Lim, C. Shu, X. D. Niu, Y. T. Chew, Application of lattice Boltzmann method to simulate microchannel flows, Phys. Fluids 14 (7) (2002) 22992308. 
[44] X. Y. He, S. Y. Chen, G. D. Doolen, A novel thermal model for the lattice Boltzmann method in incompressible limit, J. Comput. Phys. 146 (1) (1998) $282-300$.

[45] X. Y. He, L. S. Luo, M. Dembo, Some progress in lattice Boltzmann method. Part I. Nonuniform mesh grids, J. Comput. Phys. 129 (2) (1996) 357-363.

[46] L. Mieussens, Convergence of a discrete-velocity model for the BoltzmannBGK equation, Comput. Math. Appl 41 (1-2) (2001) 83 - 96.

[47] L. Mieussens, Discrete velocity model and implicit scheme for the BGK equation of rarefied gas dynamics, Math. Models Methods Appl. 10 (2000) 1121-1149.

[48] L. Mieussens, Discrete-velocity models and numerical schemes for the Boltzmann-BGK equation in plane and axisymmetric geometries, J. Comput. Phys. 162 (2) (2000) $429-466$.

[49] J. Y. Yang, J. C. Huang, Rarefied flow computations using nonlinear model Boltzmann equations, J. Comput. Phys. 120 (2) (1995) 323 - 339.

[50] K. Aoki, S. Takata, T. Nakanishi, Poiseuille-type flow of a rarefied gas between two parallel plates driven by a uniform external force, Phys. Rev. E 65 (2) (2002) 026315.

[51] K. Aoki, K. Nishino, Y. Sone, H. Sugimoto, Numerical analysis of steady flows of a gas condensing on or evaporating from its plane condensed phase on the basis of kinetic theory: Effect of gas motion along the condensed phase, Phys. Fluids A 3 (9) (1991) 2260-2275.

[52] D. Valougeorgis, Couette flow of a binary gas mixture, Phys. Fluids 31 (3) (1988) 521-524.

[53] S. Naris, D. Valougeorgis, The driven cavity flow over the whole range of the Knudsen number, Phys. Fluids 17 (9) (2005) 097106. 
[54] S. Naris, D. Valougeorgis, D. Kalempa, F. Sharipov, Flow of gaseous mixtures through rectangular microchannels driven by pressure, temperature, and concentration gradients, Phys. Fluids 17 (10) (2005) 100607.

[55] F. Sharipov, G. Bertoldo, Numerical solution of the linearized Boltzmann equation for an arbitrary intermolecular potential, J. Comput. Phys. 228 (9) (2009) $3345-3357$.

[56] F. Sharipov, D. Kalempa, Oscillatory couette flow at arbitrary oscillation frequency over the whole range of the knudsen number, Microfluid. Nanofluid. 4 (5) (2008) 363-374.

[57] L. S. Luo, Some recent results on discrete velocity models and ramifications for lattice Boltzmann equation, Comput. Phys. Commun. 129 (1-3) (2000) $63-74$.

[58] Y. B. Li, H. L. Fan, X. B. Nie, R. Y. Zhang, X. W. Shan, H. D. Chen, X. K. Chi, T. I-P. Shih, Application of a higher order lattice Boltzmann/hybrid method for simulation of compressible viscous flows with curved boundary, AIAA-2009-1336, 47th AIAA aerospace sciences meeting including the new horizons forum and aerospace exposition(2009).

[59] X. B. Nie, X. W. Shan, H. D. Chen, Lattice Boltzmann/finite-difference hybrid simulation of transonic flow, AIAA-2009-139, 47th AIAA aerospace sciences meeting including the new horizons forum and aerospace exposi$\operatorname{tion}(2009)$.

[60] K. Xu, J. C. Huang, A unified gas-kinetic scheme for continuum and rarefied flows, J. Comput. Phys., 229 (2010) 7747 - 7764. 


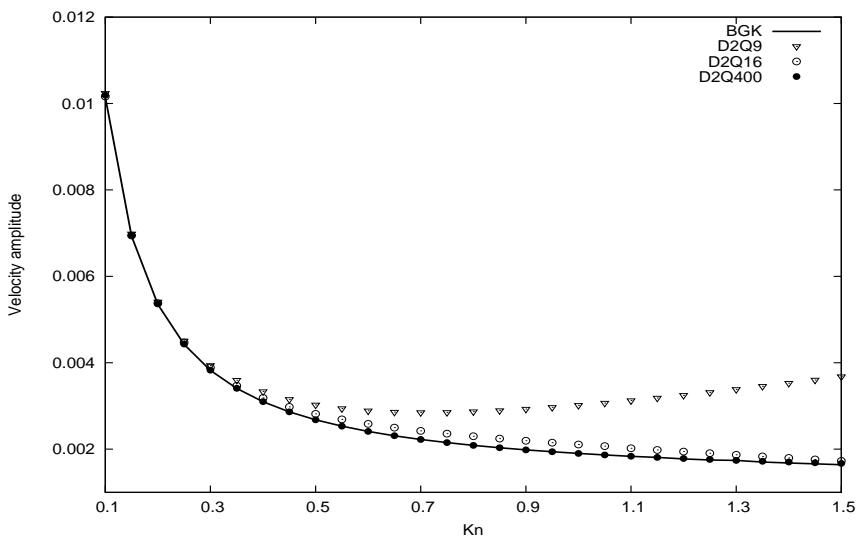

Figure 1: The results of D2Q400, D2Q16 and D2Q9 models for the quasi-steady standing shear wave. The first-order Hermite expansion is adopted for the D2Q400 model. Since the Hermite polynomials for the D2Q400 model give irrational roots, the Lax-Wendroff scheme is used to solve Eq.(13) here. The results show that the LB model with sufficiently large discrete velocity sets can be very accurate. 

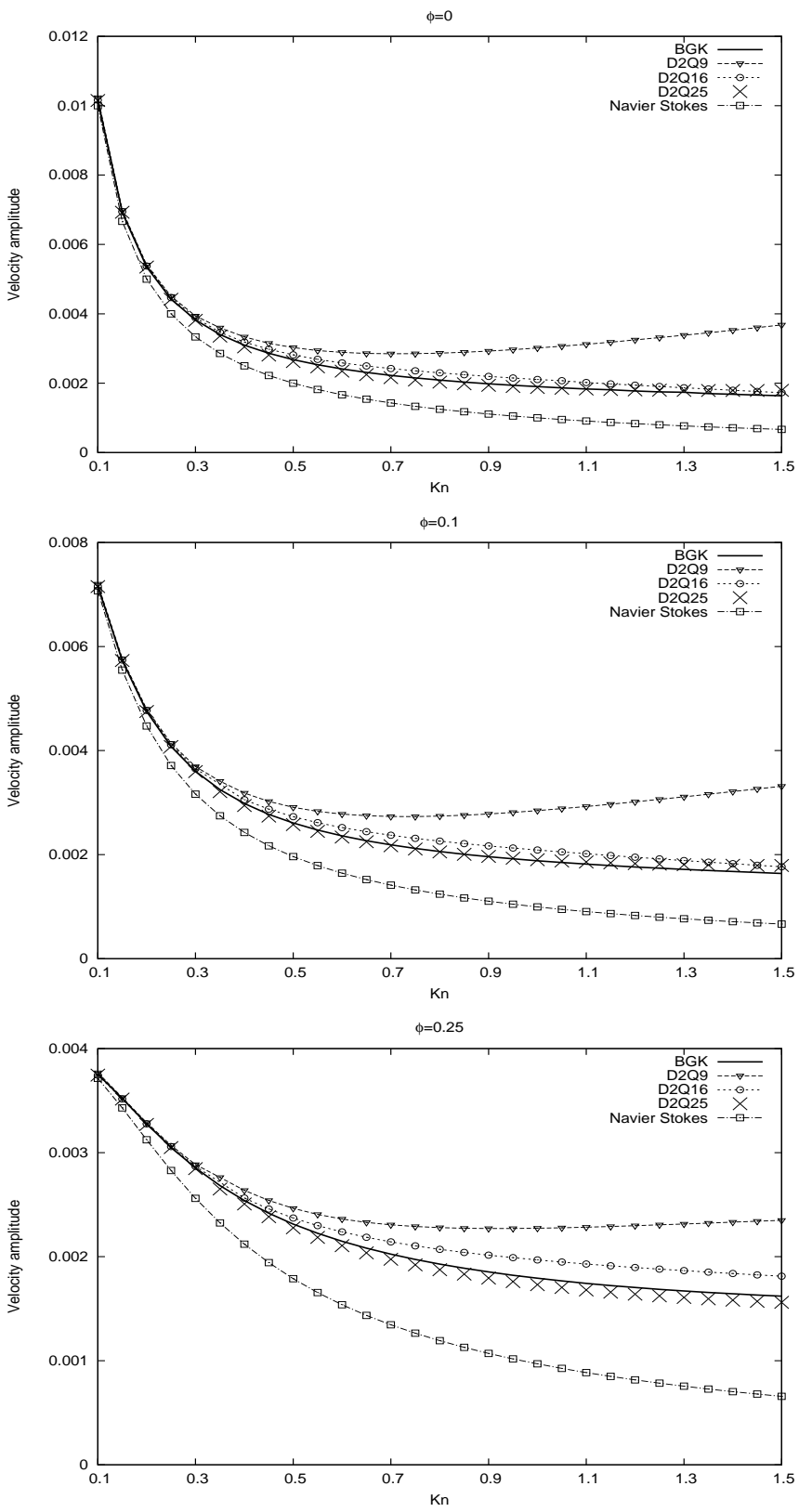

Figure 2: Velocity wave-amplitude as a function of the Knudsen number, where the expansion order for the equilibrium distribution function and the force term is $N$ which is $2,3,4$ for the D2Q9, D2Q16 and D2Q25 models respectively, and the order of Gauss-Hermite quadrature is $2 N+1$. 

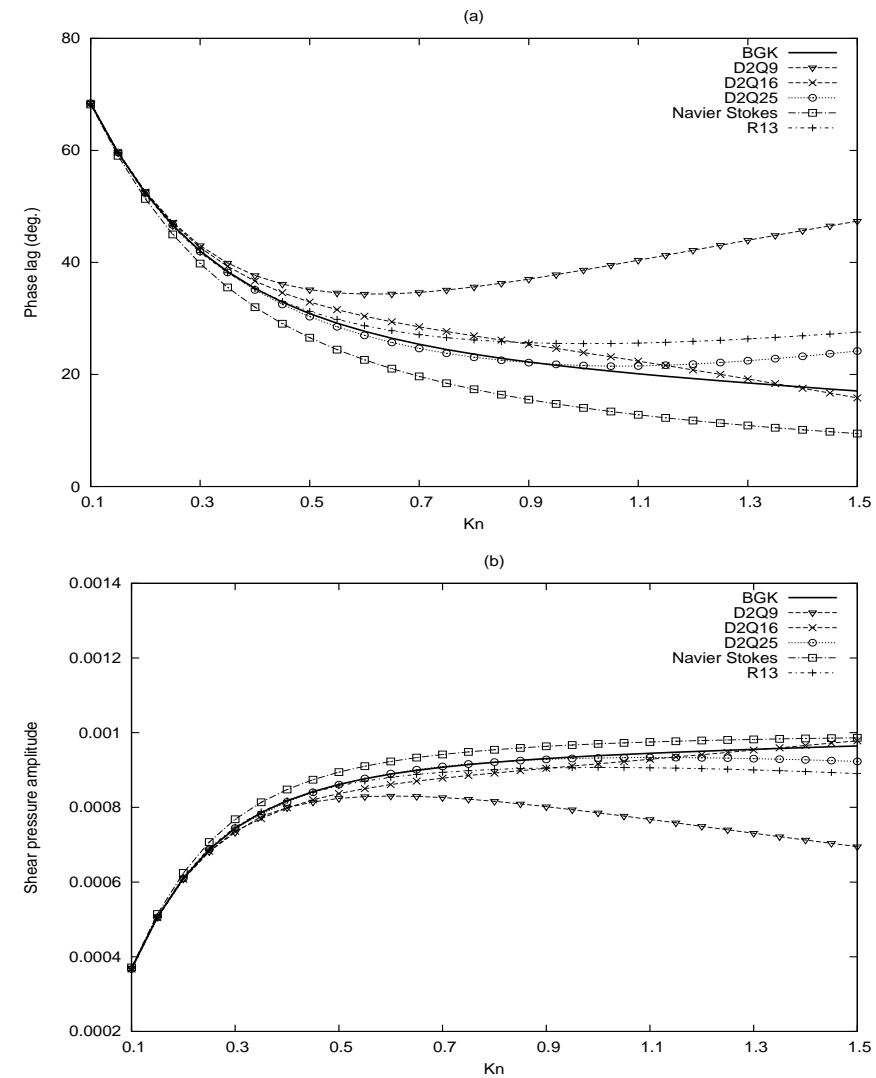

Figure 3: (a) Velocity wave phase lag, and (b) shear pressure wave amplitude as a function of the Knudsen number, where the expansion order for the equilibrium distribution function and the force term is $N$ which is 2, 3, 4 for the D2Q9, D2Q16 and D2Q25 models respectively, the order of Gauss-Hermite quadrature is $2 N+1$, and $\phi=0.25$. 

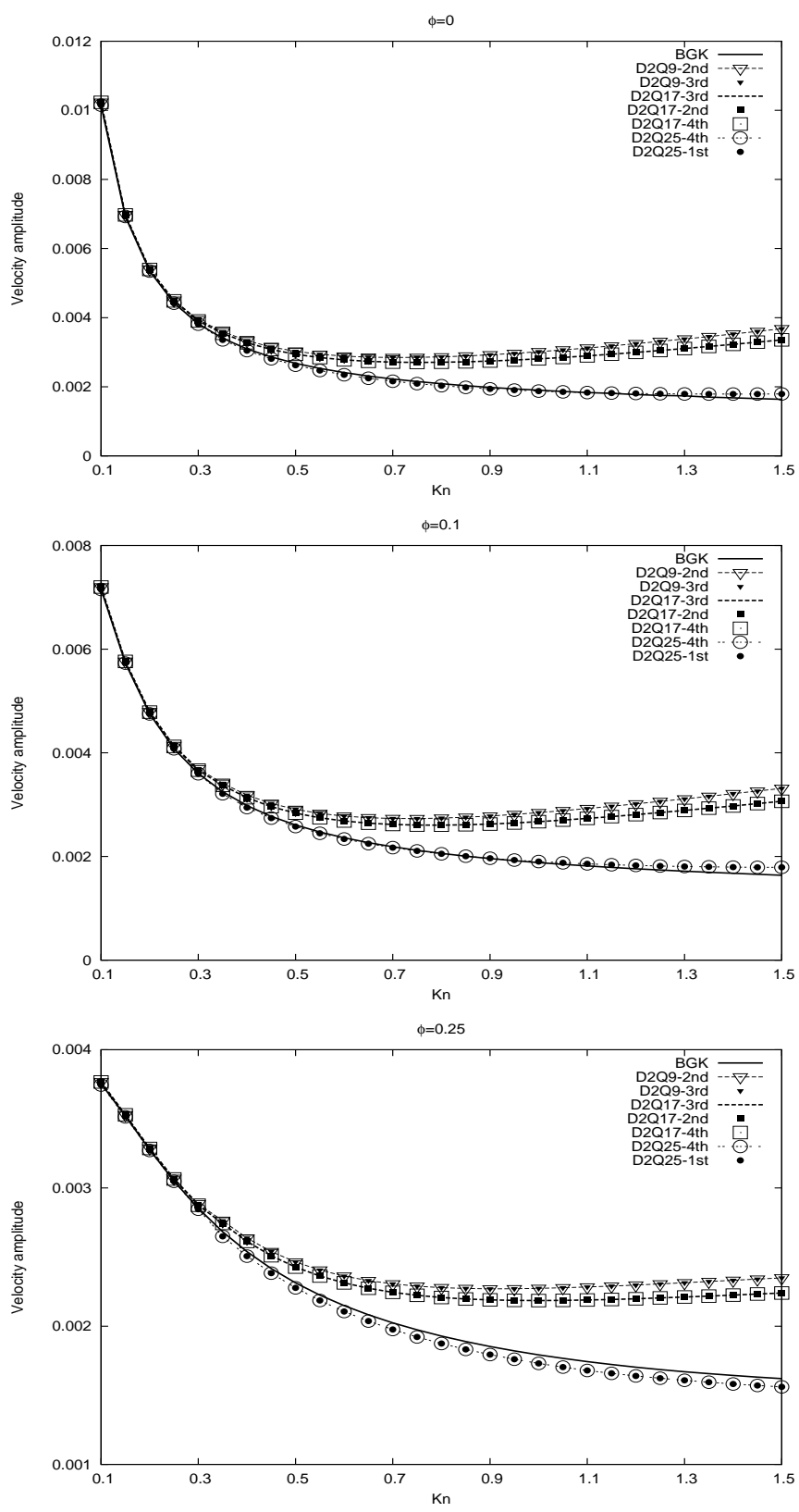

Figure 4: Velocity wave-amplitude varying with the Knudsen number. The models are named according to the rule D2Qn - Yth where n denotes the number of discrete velocities, $\mathrm{Y}$ the expansion order for the equilibrium expansion and the force term. 

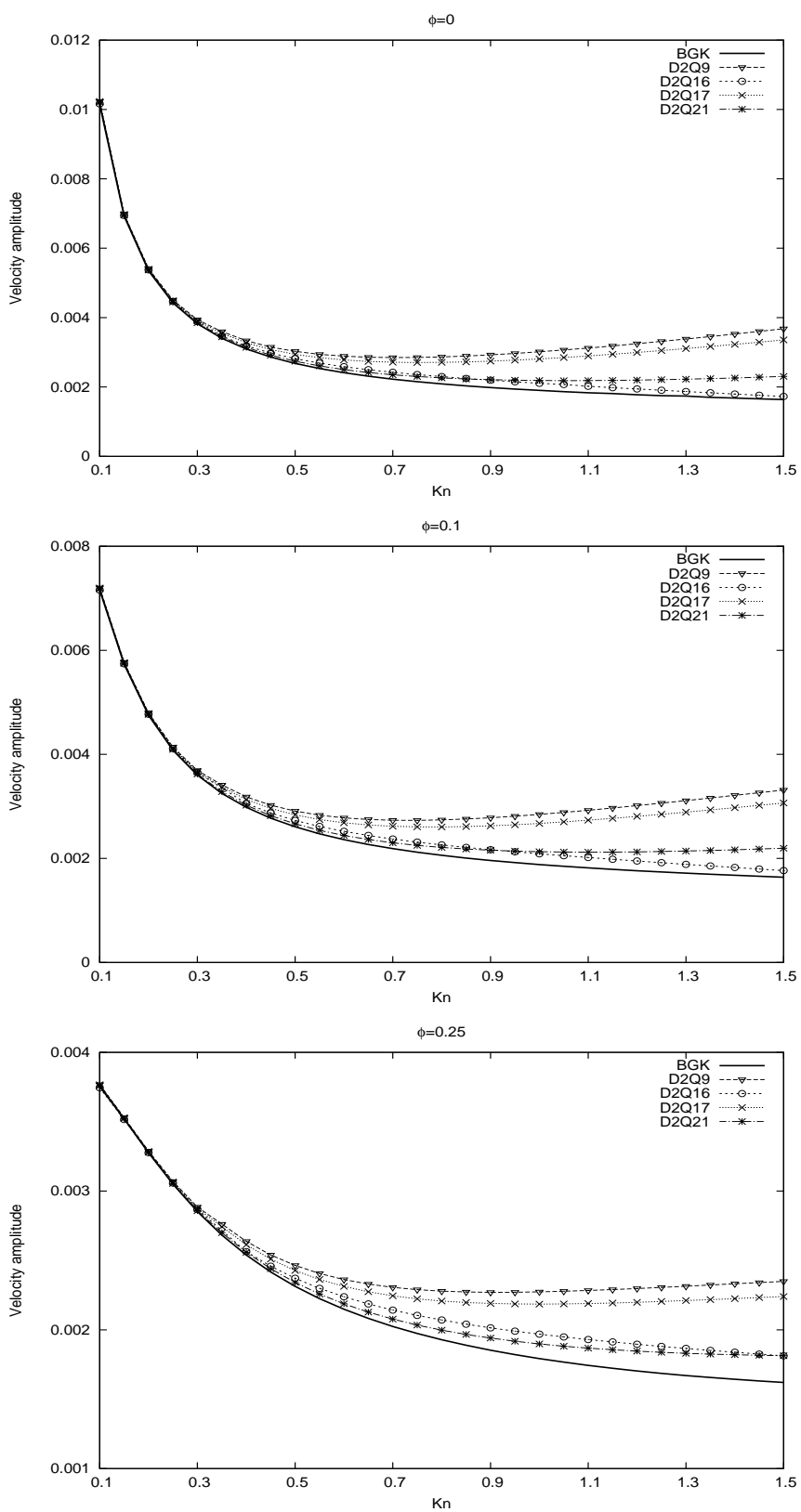

Figure 5: Velocity wave-amplitude varying with the Knudsen number, where the three models with the same order of quadratures but different abscissae are compared. 

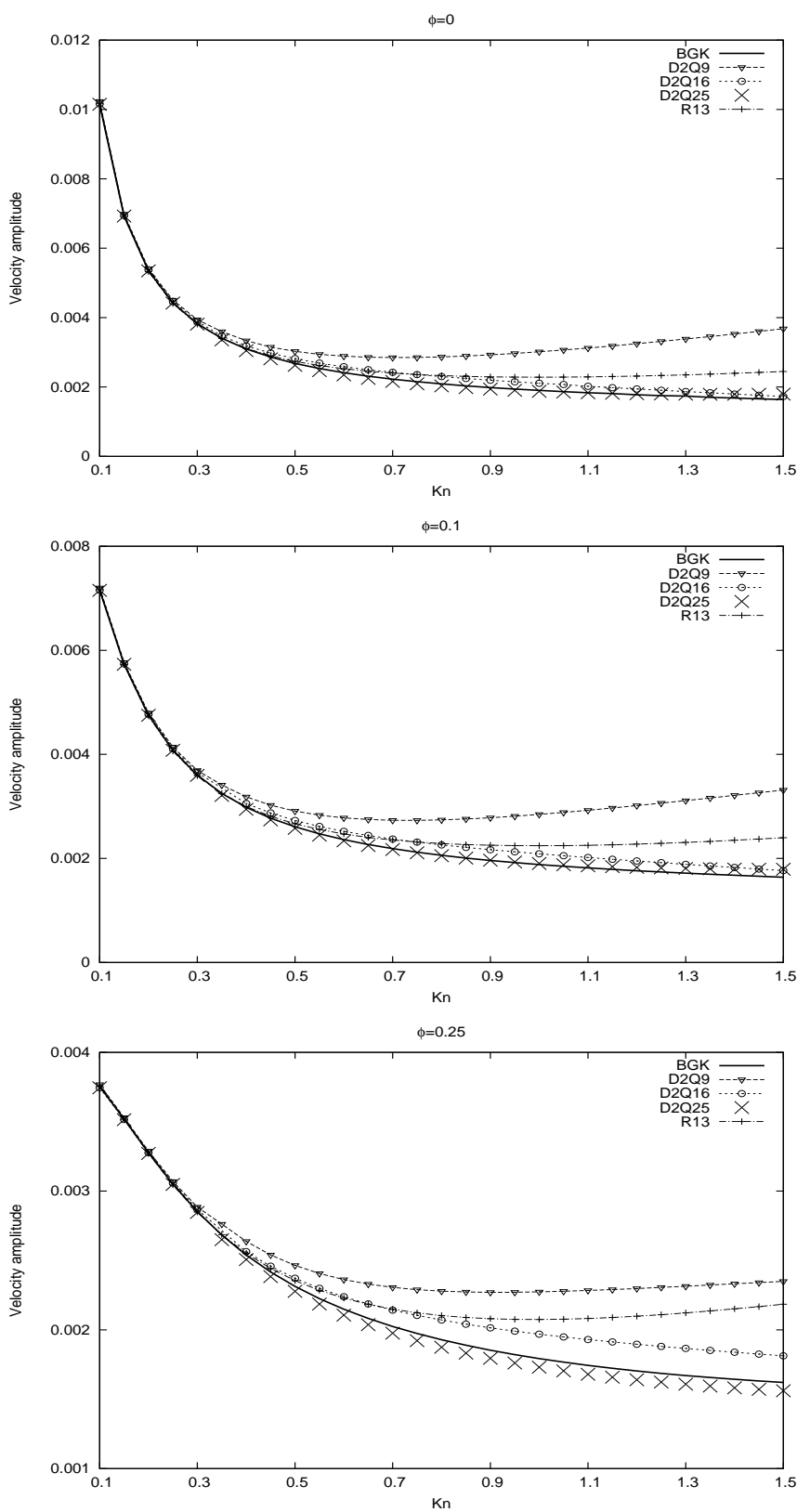

Figure 6: Velocity wave-amplitude varying with the Knudsen number where the results of LB models are compared against the solution of the R13 equation. 